\title{
Percepção dos servidores de um Hospital de Clínicas sobre os efeitos da Ginástica Laboral
}

\section{Perception of the servers from a hospital clinics in the effects of Gymnastics Labour}

\section{Percepción de los servidores de un hospital de clinicas sobre los efectos de la Gimnasia Laboral}

Recebido: $21 / 08 / 2013$

Aprovado: 06/01/2014
Isabel Aparecida Porcatti de Walsh ${ }^{1}$

Dernival Bertoncello ${ }^{2}$

Heloísa Cristina Figueiredo Frizzo ${ }^{3}$

Solanne Gonçalves Alves 4

Wellington Lourenço Mendes dos Santos ${ }^{5}$

Sabendo da importância da Ginástica Laboral como método preventivo e terapêutico, um grupo multiprofissional vem realizando a prática desta com funcionários de um Hospital de Clínicas no estado de Minas Gerais. Para avaliar a percepção dos trabalhadores sobre os efeitos da Ginástica Laboral com relação à integração com os colegas de trabalho, à disposição, ao bem-estar no ambiente de trabalho, à diminuição das dores, à conscientização sobre o corpo, à motivação para fazer exercício fora do trabalho, ao relaxamento, ao humor, à diminuição do estresse e à melhora da saúde, foi aplicado um questionário. Concluiu-se que a Ginástica Laboral trouxe grande contribuição, não apenas no que se refere aos aspectos físicos, mas também psicossociais e relacionais.

Descritores: Ginástica; Atividade Motora; Saúde.

Knowing the importance of workplace exercise as preventive and therapeutic method, a multidisciplinary group has been conducting this practice with employees of a Clinical Hospital in the State of Minas Gerais, Brazil. To evaluate the perception of workers about the effects of the same with respect to integration with coworkers, disposition, wellness in the workplace, reduction of pain, body awareness, motivation to exercise outside of work, relaxation, humor, decreased stress and improved health a questionnaire was administered. It was concluded that the workplace exercise brought great contribution, not only with regard to the physical aspects, but also psychosocial and relational.

Descriptores: Gymnastics; Motor Activity; Health.

Conociendo la importancia de la gimnasia laboral como método preventivo y terapéutico, un grupo multidisciplinario ha llevado a cabo esta práctica con los empleados de un hospital de clínicas en el Estado de Minas Gerais, Brasil. Para evaluar la impressión de los trabajadores sobre los efectos de la misma con respecto a la integración con los compañeros de trabajo, la disposición, el bienestar en el lugar de trabajo, la reducción del dolor, la conciencia corporal, la motivación para hacer ejercicios fuera del trabajo, humor , disminución del estrés y la mejora de la salud fue echa uma encuesta. Se concluyó que el uso de la gimnasia trajo un gran contribución, no sólo con respecto a la condición física, sino también psicosocial y relacional. Descriptores: Gimnasia; Actividad motora; Salud.

\footnotetext{
1 Fisioterapeuta. Professora Adjunta do Departamento de Fisioterapia Aplicada da Universidade Federal do Triângulo Mineiro (UFTM). ewalsh@terra.com.br

2 Fisioterapeuta. Professor Adjunto do Departamento de Fisioterapia Aplicada da UFTM.

3 Terapeuta Ocupacional. Professora Assistente do Departamento de Terapia Ocupacional da UFTM.

4 Terapeuta Ocupacional. Residente Multiprofissional em Saúde Mental do Instituto de Psiquiatria da Universidade Federal do Rio de Janeiro.

5 Educador Físico.
} 


\section{INTRODUÇÃO}

A realidade dos hospitais brasileiros traz a sobrecarga e a exigência por produtividade como fator estressante para seus funcionários. Muitos destes possuem um ritmo exaustivo de trabalho, inclusive com regime de plantões, carga horária de trabalho elevada, trabalho em turno noturno, mais de um emprego em razão dos baixos salários, grandes responsabilidades, risco de acidentes, atividades antiergonômicas e sedentarismo ${ }^{1}$.

Assim, de maneira geral, o hospital é considerado um recinto insalubre, penoso e perigoso para os que nele trabalham, sendo um local privilegiado para o adoecimento. Além dos riscos de acidentes e doenças de ordem física, o sofrimento psíquico é também bastante comum e está em crescimento diante da pressão social e psicológica a que estão expostos os que ali atuam $^{2}$.

Nesse contexto, há necessidade da realização de intervenções com tal população que possibilitem o desenvolvimento de um trabalho adequado, que objetive a promoção da saúde desses trabalhadores.

No Brasil, uma das formas de intervenção no trabalho mais utilizadas, com intuito de prevenção de doenças e promoção da saúde, é a Ginástica Laboral (GL), que aparece como método preventivo e terapêutico no enfrentamento de distúrbios físicos e emocionais na saúde do trabalhador ${ }^{3}$.

A GL tem como objetivos: promover a saúde, corrigir os vícios posturais, reduzir o absenteísmo e a procura ambulatorial, melhorar a condição física geral, prevenir doenças ocupacionais, controlar a dor musculoesquelética, aumentar o ânimo e a disposição para o trabalho, melhorar o condicionamento orgânico geral, prover a consciência corporal, ampliar o relacionamento interpessoal, prevenir a fadiga muscular e melhorar a qualidade de vida dos trabalhadores ${ }^{4-10}$.
A GL é a prática de exercícios, realizada coletivamente, com a finalidade de prevenir doenças ocupacionais e promover o bem-estar individual por intermédio da consciência corporal: conhecimento do próprio corpo, respeito, estímulo e amor a este e ao meio ${ }^{3}$. Consiste em exercícios adaptados para as necessidades impostas pelo tipo de trabalho, realizada sem sair do posto, em breves períodos de tempo, ao longo de todo o dia de trabalho, enfatizando o alongamento e a compensação das estruturas musculares envolvidas nas tarefas ocupacionais diárias $4,11,12$.

Este estudo tem como objetivo avaliar a percepção dos trabalhadores sobre os efeitos da GL com relação à integração com os colegas de trabalho, à disposição, ao bem-estar no ambiente de trabalho, à diminuição das dores, à conscientização sobre o corpo, à motivação para fazer exercício fora da empresa, ao relaxamento, ao humor, à diminuição do estresse e à melhora da saúde.

\section{MÉTODO}

Trata-se de um estudo analítico, transversal, de caráter exploratório e metodologia quantitativa. A amostra foi composta por trabalhadores dos setores da central de esterilização de materiais, lavanderia e centro cirúrgico do Hospital de Clínicas da UFTM que participavam do programa de Ginástica Laboral. Foram excluídos aqueles que não quiseram participar e não assinaram o Termo de Consentimento Livre e Esclarecido (Resolução n.. 196, de 10 de outubro de 1996, do Conselho Nacional de Saúde, que regulamenta pesquisas que envolvem seres humanos). 0 presente estudo foi aprovado pelo Comitê de Ética e Pesquisa da UFTM sob o n. -1.351 e desenvolvido ao longo do ano de 2010 e avaliado no ano de 2011.

As atividades do Programa de Ginástica Laboral foram realizadas durante um ano na central de esterilização de materiais, lavanderia e centro cirúrgico do Hospital de Clínicas da UFTM, duas vezes 
por semana, com a duração de quinze minutos em cada setor e conduzidas por um grupo multiprofissional composto de docentes e estudantes do curso de Fisioterapia, além de discentes das áreas de Educação Física e Terapia Ocupacional. Foram utilizadas atividades físicas e lúdicas em dupla ou em grupo, considerando-se que o lúdico é um elemento importante também na vida do adulto, uma vez que a ideia de totalidade do ser humano, independente da fase de desenvolvimento, só pode ser entendida quando inclui $\mathrm{o}$ prazer nas atividades que este realiza ${ }^{13}$. A música foi utilizada como recurso atrativo aos participantes, pois é sabido que esta proporciona alívio do trabalho e motivação a um esforço maior em determinado ritmo ${ }^{14}$. As atividades executadas variaram a cada setor de trabalho, conforme a situação do contexto físico (iluminação, temperatura, ventilação, ruído e espaço físico).

Após um ano de participação na GL, foram realizadas as avaliações, utilizandose dois questionários confeccionados para esta pesquisa. 0 primeiro foi composto de questões abertas pessoais, de hábitos e comportamentos, e teve como objetivo identificar o perfil da amostra estudada. 0 segundo questionário foi de avaliação da GL e teve como propósito levantar dados sobre esta e os resultados que trouxe para os trabalhadores na percepção destes.

Esse questionário apresentou nove questões fechadas (integração com os colegas de trabalho, disposição, bem-estar no ambiente de trabalho, diminuição das dores, conscientização sobre o corpo, motivação para fazer exercícios fora da empresa, relaxamento, melhora no humor, diminuição do estresse e melhora da saúde), respondidas por meio de uma escala visual analógica com $10 \mathrm{~cm}$ de comprimento, com as âncoras 0 e 10, em que 0 representava "não melhorou" e 10 "melhorou muito".

0 preenchimento dos questionários foi realizado em horário regular e no local de trabalho, em espaço físico apropriado, sem qualquer ônus para o trabalhador em forma de salário ou acréscimo de horas. Durante o tempo de resposta do questionário, um dos pesquisadores permaneceu no local para dirimir dúvidas.

\section{RESULTADOS}

Participaram 51 trabalhadores, sendo 42 do sexo feminino, com idades variando entre 22 e 58 anos $(40,2 \pm 10,85)$, com média de 6.9 horas de trabalho diárias. Dentre as categorias profissionais, houve 18 variações, sendo que Técnico em Enfermagem (17) e Auxiliar de Enfermagem (9) os mais presentes.

Os participantes apresentavam média de sete horas diárias de sono, sendo que 46 não eram fumantes; 20 realizavam alguma atividade física, em média três vezes por semana; e, entre os nove tipos de atividades físicas realizadas, a caminhada apareceu mais vezes (quatro).

A Tabela 1 apresenta os resultados sobre a percepção dos efeitos da GL segundo a média das respostas obtidas. Observa-se percepção positiva com relação a todos os itens avaliados, com médias superiores a oito pontos na escala de 0 a 10.

Tabela 1. Percepção sobre os efeitos da Ginástica Laboral. HC/UFTM, 2011.

\begin{tabular}{lc}
\hline EFEITOS APÓS O INÍCIO DA GINÁSTICA LABORAL & MÉDIA \\
\hline 1. Integração com os colegas de trabalho & 8,7 \\
2. Disposição e bem-estar no ambiente de trabalho & 8,7 \\
3. Diminuição das dores & 8,7 \\
4. Conscientização sobre o corpo & 8,7 \\
5. Motivação para fazer exercícios fora da empresa & 8,7 \\
6. Relaxamento & 8,7 \\
7. Melhora no humor & 9,0 \\
8. Diminuição do estresse & 8,8 \\
9. Melhora da saúde & 9,0 \\
\hline
\end{tabular}




\section{DISCUSSÃO}

Os resultados indicaram a percepção positiva dos efeitos da GL com relação a todos os itens avaliados.

Quanto aos benefícios emocionais, pode-se destacar percepção na melhora do humor, diminuição do estresse e melhora da saúde. Identificou-se também percepção positiva quanto à integração entre os colegas, disposição e bem-estar no ambiente de trabalho. Quanto aos benefícios de ordem física, foram relatados: diminuição das dores, conscientização sobre o corpo, motivação para fazer exercícios fora da empresa, relaxamento e melhora da saúde.

Outros autores também consideram as melhoras promovidas pela GL nesses aspectos. Uma revisão bibliográfica mostrou que, das evidências apresentadas por diferentes autores, ressalta-se a importância da GL no alívio das dores corporais, na diminuição dos casos de Distúrbios Osteomusculares Relacionados ao Trabalho (DORT), no aumento da produtividade e no maior retorno financeiro para a empresa ${ }^{15}$.

Um estudo 8 com trabalhadores que executavam suas funções na posição sentada, utilizando como recurso computador, mesa, cadeira, papéis, canetas e telefone durante os anos de 2002 a 2005, demonstrou que a GL realizada no primeiro ano durante 20 minutos e duas vezes por semana ao final do expediente; no segundo ano, duas vezes por semana em dois períodos (manhã/tarde); e no terceiro ano, quatro sessões semanais somente no período da manhã com a mesma duração, encontrou melhoria na disposição dos participantes, no bem-estar e na qualidade de vida destes, além da flexibilidade, da redução da incidência de dores musculares e/ou posturais durante o trabalho e ao acordar.

Um programa com trabalhadores industriais submetidos a 15 minutos de GL compensatória, cinco vezes por semana, durante seis meses, (sendo 5 minutos de aquecimento, 10 minutos de alongamento global e após atividades alternadas como fortalecimento, relaxamento, massagem e atividades lúdicas recreativas diversas), observou melhora na qualidade de vida, aumento da flexibilidade e nível de satisfação dos participantes ${ }^{16}$.

Em pesquisa realizada com 30 funcionários da linha de produção e oficina de uma indústria metalúrgica automobilística em Piracicaba, São Paulo, verificou-se que a GL pode ter contribuído para melhorar a qualidade de vida, principalmente na dimensão do estado geral da saúde ${ }^{17}$.

Numa pesquisa realizada com 40 funcionárias de serviços gerais de uma Universidade Paranaense na faixa etária de 30 a 45 anos de idade, durante seis meses, perfazendo o total de 48 sessões, foi observada diminuição da dor, melhora da qualidade de vida em relação às condições de trabalho, do relacionamento interpessoal e do estado de humor ${ }^{18}$.

Noutra pesquisa realizada com o objetivo de avaliar os efeitos da GL sobre as queixas de 24 trabalhadores de teleatendimento em uma empresa de plano de saúde em Belo Horizonte, realizada quatro vezes por semana durante 15 minutos, no período de quatro meses, perfazendo o total de 68 sessões, foram constatados efeitos benéficos sobre a saúde dos trabalhadores, diminuindo os relatos de cansaço e estresse, aumentando a disposição para o trabalho e a interação entre os colegas, além da diminuição de queixas de dor ${ }^{19}$.

Outro estudo avaliou alterações positivas na flexibilidade, no percentual de gordura e na pressão arterial com aplicação de GL durante quinze minutos, três vezes por semana, num período de quatro meses ${ }^{4}$.

Outros estudos indicam também alívio das dores corporais durante o trabalho e ao acordar, diminuição dos casos de lesões musculoesqueléticas, melhora de desempenho nos testes de flexibilidade, incentivo à prática de atividades físicas extras e aumento na percepção sobre a execução do exercício, com consequente diminuição dos casos de 
lesões musculoesqueléticas, aumento da produtividade e um maior retorno financeiro para as empresas, considerando também as melhoras do relacionamento interpessoal $20-24$.

Embora os resultados tenham identificado a contribuição da GL, os distúrbios da saúde estão vinculados ao trabalho, portanto, os programas de prevenção precisam priorizar esse aspecto e atuar levando em consideração as especificidades da atividade de trabalho. Assim, os problemas osteomusculares, o conteúdo das tarefas e o ambiente de trabalho não podem ter seus efeitos resumidos a uma única forma de prevenção, pois essa abordagem reducionista da situação de trabalho, pontual e diretiva, não considera o comportamento, as reais posturas no trabalho, bem como os determinantes das situações em que as pessoas trabalham ${ }^{21}$.

\section{CONCLUSÃO}

Os dados indicam a percepção positiva dos efeitos da GL pelos trabalhadores, identificando que esta parece ter trazido grande contribuição, não apenas no que se refere aos aspectos físicos, mas também psicossociais e relacionais.

Destaca-se que as estratégias de saúde do trabalhador devem ser mais amplas do que somente a aplicação da GL, e esta deve ser parte integrante de uma política mais abrangente na valorização da saúde dos trabalhadores, devendo-se considerar que, juntamente com a GL, deve-se implementar um conjunto de melhorias, como modificação do processo de trabalho, instituição de revezamentos ou rodízios, realização de análises ergonômicas dos postos de trabalho e adequação dos instrumentos ou equipamentos de trabalho.

Sugere-se a aplicação do mesmo questionário ao longo do tempo, para avaliar se esses efeitos são duradouros.

\section{REFERÊNCIAS}

1. Gondim KM, Miranda MC, Guimarães JMX, D’Alencar BP. Avaliação da prática de ginástica laboral pelos funcionários de um hospital público. Rev Rene. 2009; 10(2):95102.

2. Pitta AMF. Hospital: dor e morte como ofício. 5 ed. São Paulo: Annablume/Hucitec; 2003.

3. Militão AG. A Influência da ginástica laboral para a saúde dos trabalhadores e sua relação com os profissionais que a orientam. [dissertação]. Florianópolis: Universidade Federal de Santa Catarina; $2001.86 \mathrm{f}$.

4. Martins CO, Duarte MFS. Efeitos da ginástica laboral em servidores da reitoria da UFSC. Rev Bras Ciênc Mov. 2000; 8(4):913.

5. Martins CO, Michels G. Saúde x Lucro: Quem ganha com um programa de promoção da saúde do trabalhador? Rev Bras Cineantropom Desempenho Hum. 2001; 3(1):95-101.

6. Polito E, Bergamaschi EC. Ginástica laboral: teoria e prática. Rio de Janeiro: Sprint; 2002.

7. Burton AK, Balagué F, Cardon G, Eriksen HR, Henrotin Y, Lahad A et al. European guidelines for prevention in low back pain: Eur Spine J. 2006; 15(2):136-68.

8. Martins GC, Barreto SMG. Vivências de ginástica laboral e melhoria da qualidade de vida do trabalhador: resultados apresentados por funcionários administrativos do Instituto de Física da Universidade de São Paulo (Campus São Carlos). Motriz Rev Educ Fís. 2007; 13(3):214-24.

9. Carvalho CMC, Moreno CRC. Efeitos de um programa de ginástica laboral na saúde de mineradores. Cad Saúde Coletiva. 2007; 15(1):117-30.

10. Dishman RK, Dejoy DM, Wilson MG, Vandenberg RJ. Move to improve: a randomized workplace trial to increase physical activity. Am J Prev Med. 2009; 36(2):133-41.

11. Mendes AR, Leite N. Ginástica laboral: princípios e aplicações práticas. São Paulo: Manole; 2004. p. 2-16. 
12. Pinto ACCS, Souza RCP. (2004). A Ginástica laboral como ferramenta para a melhoria da qualidade de vida no setor de cozinha em restaurantes [Internet]. 2000 [acesso em 2013 ago 5]. Disponível em: http://www.deps.ufsc.br/ergon/revista/ar tigos/rita.PDF.

13. Falkenbach AP. O lúdico na visão do adulto: uma abordagem psicopedagógica. Rev Perf. 1997; 1(1):78-82.

14. Kroemer KHE, Grandjean E. Manual de ergonomia: adaptando o trabalho ao homem. 5 ed. Porto Alegre: Bookman; 2005.

15. Oliveira JRG. A importância da ginástica laboral na prevenção de doenças ocupacionais. Rev Educ Fis. 2007; (139):40-9.

16. Bertelli MB, Bodini GB, Ruas G. Efeitos da ginástica laboral compensatória na flexibilidade de trabalhadores de uma empresa de São Carlos. EFDeportes.com, Rev Digit. [Internet]. 2010 dic [acesso em 2013 ago 13]; 15(151). Disponível em: http://www.efdeportes.com/efd151/efeito s-da-ginastica-laboral-na-flexibilidade.htm. 17. Leme JACA, Meyer EC. Efectos de la gimnasia laboral en la calidad de vida de los trabajadores. CiencTrab. 2008; 10(29):100-5.

18. Santos AF, Oda JY, Nunes APM, Gonçalves L, Garnés FLS. Benefícios da ginástica laboral na prevenção dos distúrbios osteomusculares relacionados ao trabalho. Arq Ciênc Saúde Unipar. 2007; 11(2):99-113.
19. Resende MCF, Tedeschi CM, Bethônico FP, Martins TTM. Efeitos da ginástica laboral em funcionários de teleatendimento. Acta Fisiátrica. 2007; 14(1):25-31.

20. Guerra MK. Ginástica na empresa: corporate e fitness. Âmb Med Desportiva. 1995; 10:19-22.

21. Alves S, Vale A. Ginástica laboral, caminho para uma vida mais saudável no trabalho. Rev CIPA. 1999; 232:30-43.

22. Jimenes P. Ginástica laboral: bem-estar do trabalhador traz resultados surpreendentes. Rev. CIPA. 2002; 171:7081.

23. Oliveira JRG. A prática da ginástica laboral. 3 ed. Rio de Janeiro: Sprint; 2006.

24. Soares RG, Assunção AA, Lima FPA. A baixa adesão ao programa de ginástica laboral: buscando elementos do trabalho para entender o problema. Rev Bras Saúde Ocup. 2006; 31(114):149-60.

\section{CONTRIBUIÇÕES}

Isabel Aparecida Porcatti de Walsh e Dernival Bertoncello contribuíram igualmente na concepção e desenvolvimento; desenho metodológico; supervisão, coleta, tratamento, análise e interpretação dos dados, levantamento da literatura e redação do artigo;

Heloisa Cristina Figueiredo Frizzo, Solanne Gonçalves Alves, Wellington Lourenço Mendes dos Santos contribuíram na concepção e desenvolvimento, coleta, levantamento da literatura e redação do artigo. 